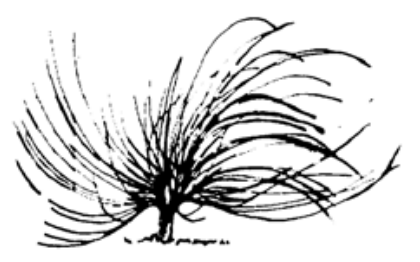

\title{
El liderazgo académico, comunicación asertiva y motivación
}

\author{
María Eugenia Ugalde Villalobos ${ }^{1}$ \\ Universidad Nacional \\ Heredia, Costa Rica \\ mgaldee@yahoo.com \\ Alba L. Canales García ${ }^{2}$ \\ Universidad Nacional \\ Heredia, Costa Rica \\ acanales25@gmail.com
}

La clave de un buen liderazgo está en su capacidad de convocar, de integrar voluntades, de escuchar y de abrir nuevos caminos. (Marchesi

y Martin 2014, p. 231)

\begin{abstract}
Resumen
Este ensayo destaca que el liderazgo es importante para mejorar el rendimiento académico, despertar el entusiasmo, la motivación, la comunicación asertiva y favorecer el trabajo en equipo, el cual lo forman docentes, estudiantes y la dirección. Además, se define cómo se ejerce el
\end{abstract}

\section{(1) 109}

Recibido: 20 de enero de 2016-Aprobado: 22 de setiembre de 2016

1 Académica e investigadora en la Escuela de Secretariado Profesional de la Universidad Nacional, Costa Rica. Trabajó como profesora de Español en el Ministerio de Educación Pública y correctora de estilo en el periódico La Nación. Bachiller en Filología Española de la Universidad de Costa Rica. Magister en Educación con énfasis en Docencia Universitaria, Universidad Nacional.

2 Académica e investigadora en la División de Educología del Centro de Investigación y Docencia en Educación, de la Universidad Nacional de Costa Rica. Licenciada en Educación Comercial, Magister en Educación con énfasis en Docencia Universitaria, Universidad Nacional. Experta en E-learning de la Fundación FATLA. 
liderazgo actualmente, por qué es importante, cuál es su relación con el contexto educativo y cómo se relacionan la comunicación asertiva, la escucha y la motivación con el liderazgo académico, elementos importantes para una mejor gestión académica.

Palabras clave: Liderazgo académico, motivación, comunicación asertiva, escucha, contexto educativo, trabajo en equipo.

\begin{abstract}
This essay emphasizes that leade ship is a key factor for improving academic performance. This is so because leadership sparks excitement, motivation, assertive communication, and it fosters teamwork among teachers, students, and the principal. Further, this essay explains how leadership is currently provided, why it is important, and how it relates to the educational context. Finally, it explains how assertive communication, listening, and motivation are related to academic leadership as these key elements for an improved academic management.
\end{abstract}

Keywords: academic leadership, motivation, assertive communication, listening, educational context, teamwork

\title{
Introducción
}

El siguiente ensayo contiene los temas comunicación asertiva, escucha, motivación y liderazgo académico, los cuales son aspectos fundamentales del liderazgo. De acuerdo con Gross (2013):

El liderazgo es el resultado de personas con un conocimiento y un talento determinado, que se ven impulsadas por la visión de lo que quieren que la organización sea, no obstante, de herramientas educativas que les permitan mejorar lo que ya poseen y adquirir los que le complementa. (p.15)

Asimismo, las herramientas por contemplar más adelante son la motivación y la comunicación dentro del ámbito educativo. Por otra parte Luna (s. f.) plantea: 
Los líderes son efectivos solo cuando cuentan con el apoyo de personas que comparten sus ideas y se comprometen a trabajar para el cambio de la realidad; los cambios propuestos por el mismo deben transcender a la persona que lidera incluso a la organización a la que pertenece, alcanzando de igual manera a las personas a quienes está dirigido al servicio que presta la organización. (p.1)

Según la cita anterior, el líder es una persona que trabaja para cambiar la realidad, por lo tanto, se refleja en el educador o educadora dentro del aula, dentro de la institución y dentro de la sociedad. Lo mismo sucede con el director o directora de un centro educativo, quien debe ejercer un liderazgo sobre todo a nivel de motivación, escucha y comunicación asertiva para que se vea el cambio.

\section{Comunicación asertiva y liderazgo}

En el siglo XXI, el asunto de la comunicación asertiva es un desafío fundamental para que un equipo trabaje de la mejor manera. Si esta situación no se cumple, siempre habrá grandes conflictos dentro de la institución educativa, por ejemplo, si el personal académico estuviera en desacuerdo con las propuestas de ls dirección. Por eso, la comunicación es esencial en el contexto educativo.

Por otra parte, la comunicación es un elemento importante del liderazgo para que haya respeto, tolerancia, integridad, con el fin de apoyar a las demás personas en la institución educativa y debe estar presente en el personal docente y estudiantes; entre el dirección, docentes y estudiantes para un mayor éxito en el quehacer académico de la institución. Actualmente, el liderazgo está distribuido en la comunidad educativa, con el propósito de acompañar, orientar, mejorar el centro educativo, es decir, todo el personal trabajando para un mismo fin. Docentes en el aula frente a su alumnado y el director o la directora deben ser líderes para docentes y para el estudiantado. Sin embargo, a veces esas premisas no se cumplen.

Elizondo, (1999) plantea:

La asertividad es la habilidad de expresar tus pensamientos, sentimientos y percepciones, de elegir cómo reaccionar y de hablar por 
tus derechos cuando es apropiado. Esto con el fin de elevar tu autoestima y de ayudarte a desarrollar tu autoconfianza para expresar tu acuerdo o desacuerdo cuando crees que es importante, e incluso pedir a otros un cambio en su comportamiento ofensivo. (p.17)

Según Llorente (2016 p.1), "la habilidad para interactuar con los demás tiene algunos trucos, como el elogio, la petición de respeto, expresar sin herir, cuando la otra persona insiste, cuando la otra persona nos ha agredido sin mala intención y cuando nos ataca". Por ejemplo, es importante que personal docente y directivo se interesen por sus estudiantes con frases como: se siente bien, cómo siguió su mamá..., me gustó mucho su presentación, muy buen trabajo, etc. A veces se olvida elogiar el esfuerzo y señalar los detalles positivos. Sin embargo, a toda persona le gusta que le digan palabras agradables y que le valoren el trabajo realizado.

Según el periódico Al Día, del 2 de julio del 2010, en Costa Rica sucedió un caso drástico en un centro educativo: el asesinato de una directora por parte de un estudiante. Si hubiera habido un ambiente de confianza, respeto, valor por la vida, la motivación, la buena comunicación, la realidad hubiera sido otra. De lo anterior se deduce que quien enseña influye de manera tanto positiva como negativa en la formación integral de las futuras generaciones y esto le compromete de manera definitiva.

La relación de liderazgo con el contexto educativo se presenta en varios ámbitos. En primer lugar, está el dedocente y estudiante. Owens (1976, citado por Elizondo, 1999) enfatiza:

Los años de formación son para el estudiante un reto constante y presentan un sinnúmero de tareas y cuestionamientos. Seres que actúan de manera fundamental en el planteamiento de dichos retos y en el cumplimiento de los objetivos, son cada uno de los maestros que interactúan con el alumno y le trasmiten conocimientos y experiencias. (p. 32)

Lo anterior se relaciona con el liderazgo académico, porque el personal docente debe ser un líder en el proceso de formación del alumnado, en donde se oriente y se compartan experiencias fundamentales para el buen desenvolvimiento estudiantil. Eso se logra, según Elizondo (1999), a través de conductas asertivas como sentirse libre de 
manifestarse a través de palabras y actos que declaren: "este soy yo", "de esta manera siento y pienso". Poder comunicarse con personas de todos los niveles dentro del ambiente educativo (compañeros y compañeras de clase, personal asesor, docente y tutor), en una forma abierta, directa y franca.

Como lo indica Civila, en Liderazgo y educación “... el liderazgo moral está caracterizado por un espíritu de servicio, solidaridad y respeto, lo que contribuye al enriquecimiento de todas y todos" (2013, p. 129). Es decir, al haber una buena relación entre docente y alumnado habrá mejor comunicación, rendimiento, eficiencia, responsabilidad, motivación en un proceso de enseñanza aprendizaje en donde se manifiesta al ente docente como una persona que trabaja por el bienestar de sus estudiantes. El liderazgo educativo está netamente relacionado con la eficiencia y eficacia del centro educativo.

Por ejemplo, una de las autoras desarrolló una actividad en la cual un grupo de estudiantes realizó una gira a un colegio rural, en setiembre de 2015: allí se observó que mientras esperaban que iniciara la clase, se pusieron a jugar dominó en el pasillo. La impresión fue interesante, porque se consideró que aprovechaban el tiempo en una actividad sana, en donde podían compartir, se divertían mientras llegaba la hora de la clase; asimismo, en algunas aulas se veía a docentes revisando trabajos, algunos casos en compañía de estudiantes, mientras el resto del grupo esperaba su turno, comentaba acerca de la materia $u$ otros temas. El escenario parecía entusiasta, saludable, estudiantes "buenos y buenas", en el sentido de que no se escuchaban malas palabras ni falta de respeto hacia las demás personas, había tranquilidad; lo mismo sucedía en los pasillos o en las zonas verdes donde descansaban o esperaban que llegara la hora de ingresar a otras clases. Sin embargo, al llegar a Heredia se reflejó un escenario contrario a la zona rural: por una acera venían unos alumnos y el lenguaje que utilizaban era de malas palabras y una percepción de aburrimiento.

Una verdadera misión justifica un gran esfuerzo, un gran trabajo docente como líder en la clase, con su ejemplo de acompañamiento, solidaridad, incluso un gran sacrificio personal o de un equipo. No importa que sea pequeño, pero sí que se perciba como relevante. Por ejemplo, una de las suscritas trabaja con estudiantes quienes deben llevar cursos de redacción y la docente todas las semanas invierte gran cantidad de tiempo revisando sus escritos con el fin de que haya aprendizaje, porque 
si el estudiantado no redacta ni la docente revisa, entonces ninguna de los dos partes sabrá si hubo o no aprendizaje.

Sin embargo, ella no ve el trabajo como sacrificio, porque se preocupa por el buen rendimiento de sus estudiantes, quienes le responden con las redacciones porque el escribir es un proceso de práctica, sin escribir no se aprende. En este caso, la satisfacción más grande para la docente, es que sus estudiantes le digan: "profesora como he mejorado mi redacción". El hecho de que a un estudiante le cueste mucho redactar, pero con la práctica supere el problema, constituye una satisfacción que no tiene precio para la docente, aunque las revisiones le hubieran implicado gran cantidad de tiempo.

Además, para que haya liderazgo académico, el personal docente debe estar convencido de que el trabajo que realiza con sus estudiantes es una labor fructífera y debe hacerla lo mejor posible: se debe pensar en que se trabaja por vocación y no por un salario. Cada docente debe estar al servicio de sus estudiantes y de la sociedad. Los educadores y las educadoras deben ser personas que se sientan satisfechas con su trabajo.

Sin embargo, no solo debe haber una comunicación asertiva entre docente y alumnado sino también entre el director o directora, docente y alumnado. Si esta relación se cumple a cabalidad, habrá mejores relaciones interpersonales entre el cuerpo docente, el administrativo y el estudiantado.

Marchesi y Martin (2014) manifiestan que una de las situaciones que acompañan ahora al alumnado es la crisis económica en sus hogares, por lo que es necesario tomar en cuenta las demandas, sus expectativas y sus exigencias, dado a que muchas veces se pretende cambiar la escuela, sin escuchar y atender sus mensajes.

El personal directivo o docente es un líder porque debe promover nuevas soluciones a los problemas que aquejan a su alumnado y velar por mantener una estabilidad dentro del aula, de tal manera que el proceso de enseñanza y aprendizaje sea significativo y ofrezca mayores oportunidades para la vida de las nuevas generaciones.

Otro aspecto importante por tomar en consideración es la comunicación asertiva; cuando existe interés entre el sujeto receptor y el sujeto emisor se produce la empatía y el rendimiento, tanto de la parte docente como de la estudiantil, mejora. Además, si se suma la asertividad del director o directora se logra un trabajo en equipo de calidad. La asertividad es la actitud que tiene una persona al expresar su punto 
de vista de un modo claro y de una forma totalmente respetuosa ante la persona interlocutora.

Si se relacionan con el liderazgo académico, en quien se dedica a la educación debemos encontrar esas mismas características, porque la profesión docente es sobre todo una vocación, como se mencionó anteriormente. Una vocación de ayuda, orientadora, social y capaz de llenar de sentido a cualquier docente. El ejercicio del liderazgo, en esa vocación docente, es un aprendizaje (García, 2005).

De acuerdo con García (2005), el profesorado competente habla de conocimientos pero sobre todo de habilidades didácticas de comunicación, de entusiasmo, de motivación, de interacción con la clase y con cada estudiante y de autoridad moral. Según la cita anterior, si el personal docente posee entusiasmo e interactúa con el estudiantado se ubica dentro del liderazgo transformacional, el cual está muy relacionado con el académico, porque las características tanto de uno como de otro son esenciales en un centro educativo.

Actualmente, el nuevo profesorado, bien liderado por una buena dirección del centro, comparte una vocación y una misión educativa valiosa, y es la única alternativa razonable para una educación significativa. Marchesi y Martin (2014), indican que:

las administraciones educativas... han de velar para que los profesores cumplan con sus obligaciones y mejoren sus enseñanzas pero también deben comprender la situación de cada uno y respetar su autonomía pedagógica; han de promover la colaboración y el trabajo en equipo en ambientes donde prima el individualismo y la falta de tradición para la cooperación; han de ampliar la participación de la comunidad educativa y de otros sectores sociales... han de buscar mantener el ánimo, la moral y la iniciativa de la comunidad educativa, en estos tiempos de recortes económicos, de presión hacia el profesorado y desanimo generalizado. (p. 205).

El mismo autor indica:

El liderazgo instruccional es el que produce efectos más positivos, es decir, mayor colaboración entre los profesores, mayor reconocimiento de la participación en el desarrollo profesional 
de los docentes y de sus prácticas innovadoras; tendencia a desarrollar programas de formación para los profesores menos competentes; y mejores relaciones entre los profesores y los estudiantes. (p. 222)

\section{Escucha y liderazgo}

Según Elizondo (1999):

En la comunicación asertiva, la habilidad de escuchar es básica para el éxito del proceso comunicativo, y para el desarrollo armonioso de las relaciones interpersonales. Dentro del salón de clases, permitirá determinar aspectos como las dudas concretas, las inquietudes, los elementos que generan discusión, los temas que causan conflicto y las posibles formas de solución al mismo, etc. fuera del salón de clases la actividad de asesoría e incluso de un tutoreo de calidad. (p. 103)

Lo anterior se relaciona con el liderazgo, ya que para que haya comunicación asertiva se debe saber escuchar. La comunicación se presenta solo si hay escucha. La mayoría de conflictos se resuelven, si se escucha detenidamente a la otra persona. En este caso, puede ser una estudiante, un docente, una directora, un padre de familia o un miembro de la comunidad y se presenta en todos los niveles: materno, preparatoria, primaria, secundaria y universitario. Se debe escuchar para ser empático.

Roberto Bell (1987, citado por Elizondo, 1999) argumenta:

La empatía es multifacética, involucrando lo cognitivo (pensamiento), lo afectivo (sentimiento) y los componentes de la comunicación. Cognoscitivamente, la persona empática toma la perspectiva de otra persona, y al hacer esto se esfuerza para ver el mundo desde otro punto de vista. Afectivamente, la persona empática experimenta las emociones de la otra; él o ella siente las experiencias del otro. Comunicativamente, el individuo empático muestra comprensión e interés a través de claves verbales y no verbales. (p. 108) 
Todos los elementos que se mencionan en la cita anterior son indispensables en la actividad de escuchar, por consiguiente, el liderazgo académico está relacionado con la escucha y la empatía.

Por ejemplo, un docente debe escuchar cuidadosamente a sus estudiantes, comprender sus sentimientos, estar interesado en lo que dicen y ser sensitivo a sus necesidades. Es importante aclarar que no siempre se va a estar de acuerdo conel estudiantado, verbigracia, un estudiante que no presentó la tarea por razones fuera de su control, y que continúa con la misma explicación en otras tareas, durante el ciclo, se le debe comunicar que no puede seguir con la misma situación, pues tuvo el tiempo estimado para entregar las asignaciones. Todas esas características están relacionadas con el liderazgo transformacional, el cual se asemeja al que se debe ejercer en el centro educativo cuando el director o directora se relaciona con el personal docente o en el aula cuando este escucha a sus estudiantes.

\section{Motivación y liderazgo}

Para que haya buena calidad educativa, debe haber una relación muy estrecha entre motivación y liderazgo. Según Cortés (2004), en investigaciones precedentes se ha logrado determinar que existe una relación muy directa entre el liderazgo y la motivación de los trabajadores y las trabajadoras. Por eso, este vínculo entre ambos factores parece prevalecer sobre otro tipo de variables (entre estas se encuentran, por ejemplo, las condiciones ambientales o sistemas de incentivos y su respectiva en relación con la motivación).

Se puede comprender que la motivación laboral está asociada con el liderazgo que se ejerce sobre esa persona, pues el estilo de liderazgo es el que va a proveer el ambiente propicio para la elección personal, sin que existan coerciones o castigos posteriores. Cuando se habla de coerciones y castigos, se refiere al liderazgo transacional. En un centro educativo no debe haber este tipo de actitud, porque eso aminora el rendimiento académico, la motivación y la comunicación.

Según Cano (1998, citado por García, 2005), uno de los factores fundamentales para el logro de una educación de calidad es el liderazgo del equipo directivo de la institución. Es necesario un liderazgo visible de la dirección, un liderazgo eficaz basado en el compromiso con la calidad, cultura en la que el líder ha de hacer partícipe a todos los 
miembros de la comunidad educativa. Por eso, para que esa situación se cumpla debe haber motivación del alumnado, hábitos y actitudes ante el trabajo intelectual, actitudes intelectuales, nivel del profesorado, modelo de enseñanza, trabajo en equipo, clima institucional, lo cual conlleva a la satisfacción estudiantil.

Así mismo, Cano plantea que se debe cambiar para tener una preparación académicamente en forma y para ser más eficaces en el logro de los objetivos como personas, comunidades e instituciones. Además, dice:

No es con excusas, arguyendo falta de condiciones óptimas, lo que va a mejorar la educación costarricense, sino la actitud positiva y el liderazgo efectivo de cada uno de nosotros. Los que hemos tenido la oportunidad de laborar en las aulas de este país allende nuestras fronteras en zonas urbanas y rurales, en todos los niveles del sistema educativo, sabemos que nunca vamos a tener esas condiciones óptimas, por eso debemos escudarnos en este tipo de excusas para negarnos la oportunidad de tener éxito. (p. 17)

Por otra parte, están aspectos como las relaciones humanas, la escucha, atención de quienes conforman el salón de clases y la institución en general, fuentes como docente y estudiante, que puedan convivir y salir adelante. Si en el muchacho que le disparó a una directora hubiera habido motivación, no hubiera sido capaz de realizar ese acto delictivo porque hubiese pensado en las consecuencias negativas que esa actitud le traería a él, a la familia, a la institución, a los compañerosy compañeras y a la directora.

Por consiguiente, en el rol docente se debe trabajar no solo el área de los conocimientos, las habilidades, las destrezas, las actitudes, sino también aspectos como la motivación y la comunicación, pilares fundamentales para que haya buenas relaciones interpersonales no solo en un centro educativo sino también dentro de la sociedad.

Lo anterior da pie para que mucho personal de educación se queja del ambiente laboral, de los sueldos, de la cantidad de estudiantes, de su mal comportamiento, del director o directora, de que el Ministerio de Educación Pública no le da capacitación, de que no hay equipo tecnológico, de falta de material didáctico, del desinterés del estudiantado, entre otros aspectos. Sin embargo, no se hace nada por mejorar algunas de las situaciones. 
Por ejemplo, en una experiencia de una de las autoras en un curso virtual, un grupo de docentes se quejó de que ahora el estudiantado, por medio de mensajes de texto, escribe muy mal y nadie hace nada para mejorar esa situación. Entonces, en una de las participaciones se hace énfasis en que es tarea de todo el profesorado buscar alternativas, velar porque sus estudiantes puedan ir corrigiendo esas faltas, tomar la iniciativa de insistirles en que en los exámenes, los trabajos extraclase y en el cuaderno se debe escribir correctamente. Por eso, cuando se les permite usar frases incompletas y con errores ortográficos, se les hará costumbre y se perderá el buen uso del lenguaje. Por otra parte, no soloel personal docente de Español debería apoyar más esta situación, sino todos y todas; pero a conciencia. No deben esperar a que el Ministerio de Educación Pública los inste a velar por el tema de la redacción y ortografía. Cada quien debe velar por hacer su trabajo lo mejor posible, aunque haya con pocos recursos. Si al estudiantado se le exige de una manera asertiva, da buen rendimiento.

Gutiérrez (2005) plantea que actualmente se debe aspirar a una educación que, como lo afirma la escritora Lidia Clara Román, nos eduque para entender que: "Con dinero podemos comprar una cama, pero no el sueño. Libros, pero no cultura. Comida, pero no belleza. Una casa, pero no un hogar. Medicinas, pero no salud. Lujos, pero no simpatía. Diversiones, pero no felicidad. Un crucifijo, pero no un Salvador. Una iglesia,pero no el cielo. Una educación que nos recuerde permanentemente que 'lo que el dinero no puede comprar, Dios lo da diariamente sin cobrar"” (p. 18).

Actualmente, la tecnología invade a muchos y muchas estudiantes, quienes quieren tener el último modelo de teléfono celular, lo que muchas veces los lleva a hacer grandes gastos, sin pensar en que pueden existir aspectos más importantes que tener un teléfono último modelo. Otra alternativa en donde el estudiantado puede aportar a la sociedad $\mathrm{y}$, por ende, en su crecimiento personal, son los proyectos educativos como las giras, trabajos de bien social, visitar un asilo de personas adultas mayores, un colegio rural, una comunidad indígena, un grupo de adultos y adultas mayores, entre otros. En esas experiencias, el estudiantado sentirá que puede apoyar con sus iniciativas, los conocimientos adquiridos en la universidad. De esa forma, se va creando conciencia, valores, trabajo colaborativo, lo cual les hace mejores personas. El personal de educación siempre debe cultivar, en sus estudiantes, el 
servicio hacia las demás personas, porque las mayores satisfacciones se adquieren con comunicación, escucha y motivación ante los retos que se presentan a lo largo de la vida.

Los educadores y educadoras son líderes porque tienen la responsabilidad de formar los futuras generaciones constructoras de la nacionalidad costarricense, no las destructoras de los esfuerzos e ideales del resto. Destruir es fácil. Construir es mucho más difícil. Para lograrlo no hay que tener miedo, pero sí carácter (Gutiérrez, 2001).

Para ilustrar la cita anterior, una de las suscritas vivió una situación un poco desmotivante cuando una docente dijo en la sala de profesores: "háganle bastantes boletas a 'fulanito' para que se quede". Ahí se nota la falta de vocación de esa persona, ya que en vez de ayudar más bien lo que trajo como consecuencia fue que el muchacho desertara del sistema educativo. De esa manera, no se construye a nadie más bien se destruye. No se motiva, sino que se desmotiva. En esa "educadora" no hay liderazgo porque el líder ayuda, transforma, motiva, forma personas críticas para la sociedad.

También se dan casos en los cuales los profesores y profesoras motivan a sus estudiantes. Cuántas veces se ha escuchado en estudiantes que dicen "por esa o ese profesor estoy estudiando, por él o ella soy lo que soy". Lo anterior se puede relacionar con una experiencia que le comentaron a una de las autoras acerca de una profesora que trabajó en el colegio Los Guidos de Desamparados y un grupo de estudiantes tenía un vocabulario cuyo argot era propio de la delincuencia, otra parte llegaba sucia a la clase, pero la docente fue colaborando para que cambiaran, por eso cuando se acercó el día de la graduación, un estudiante le dijo que no iría y ella le preguntó por qué: él le contestó que no tenía ropa para ponerse ese día. Dicha profesora tenía dos hijos de esa misma edad, pensó que la ropa de uno de ellos le serviría, por lo tanto, el muchacho muy contento le agradeció y pudo asistir.

De lo anterior, se puede rescatar que un buen líder, además de motivar, se interesa por sus estudiantes, se preocupa por su bienestar, busca soluciones y apoya.

Plantea el Informe sobre desarrollo humano (PNUD, 2015, p. 2), que "son las personas las que cuentan, hay que influir en el proceso de desarrollo que determine sus vidas, de manera positiva, el crecimiento económico es un medio importante, pero no es la meta última". 
En consecuencia, no solo en el plano educativo se plasma el liderazgo y la motivación, por ejemplo, muchas veces, algunas personas se preocupan por el bienestar de otras, tal es el caso de cuando surgen desastres naturales como terremotos, inundaciones o derrumbes.

La motivación está relacionada con el liderazgo académico, porque el personal docente es el precursor de conseguir la calidad en todos los ámbitos. Además, si motiva, se le hace más fácil facilitar del aprendizaje. Si hay motivación, habrá calidad.

Por otra parte, "debemos tener presente al hablar del costo de la educación que el fracaso y frustración de nuestros jóvenes puede salir más caro que la excelencia, por eso debemos tener presente como lo hicieron nuestros antepasados, que invertir en una educación inclusiva de calidad no es gasto sino una inversión en el desarrollo del país" (Gutiérrez, 2005, p. 19).

Si se trabaja con liderazgo, se tiene en cuenta que en momentos de crisis es necesaria la energía, la fuerza, el amor, los principios, el respeto, la solidaridad. El amor es acción. Es ayuda, es esfuerzo y dedicación y eso implica el liderazgo. Es cooperación. No es desear el bien sino ayudar. Es aplicarle un examen a un estudiante sin la necesidad de pedirle un dictamen médico porque tal vez ese día no tenía dinero para los pasajes. Es integridad física, social y espiritual. Todo ello se consigue cuando se trabaja con dedicación, compromiso, motivación y esfuerzo.

La motivación es un desafío del siglo XXI no solo en un centro educativo sino también para docentes, personal directivo y estudiantes, porque se vive en una sociedad llena de violencia, drogas, corrupción, problemas económicos y de comunicación que afectan al estudiantado. Si este está motivado, se creará un ambiente respetuoso, comprensible y con gran disposición para estar en el aula.

Cuando se presenta una actitud de motivación, esta se contagia. Por ejemplo, una de las autoras vivió una experiencia en un centro educativo, hace dos años: un grupo de estudiantes se inscribieron en un torneo futbolístico a nivel nacional cuyas personas ganadoras irían a Brasil. Entonces, contaban a sus docentes "vamos para Brasil". Lo anterior indica que tenían mucha motivación por el torneo futbolístico.

Otro requisito importante, durante una lección, es tener presente el currículo oculto, el cual tiene mucha relación con la motivación y algunos valores. Como experiencia de una de las autoras, una vez un muchacho en un centro educativo perdió todo por causa de los fuertes 
aguaceros, el río aumentó mucho su caudal y arrastró una casa con todo lo que tenía adentro. Esa casa era de muy mala calidad porque estaba construida en un precario y la familia quedó sin nada, inclusive sin uniformes ni cuadernos. Por consiguiente, cuando la profesora explicó la situación, su grupo le trajo víveres para ayudarlo, por lo que reinó el espíritu de servicio, solidaridad y compañerismo.

El tema del liderazgo académico encierra muchos factores no solo para el profesorado o maestros y maestras, sino también para directoras y directores. Gutiérrez (2001) apunta:

Tengan presente que el líder sirve de guía, promueve la confianza, el entusiasmo y la cooperación de tal manera que aúna esfuerzos con sus colaboradores y los hace partícipes de su visión y compromiso por hacer realidad conjuntamente la misión y los objetivos institucionales. En este sentido, cuando hay errores, no busca culpables, sino que corrige esos errores sin atacar personas; su meta es resolver problemas y superar obstáculos. No define a sus subalternos como tales, sino como colaboradores y seguidores. (p. 42)

Por otra parte, Bennis (citado por Gutiérrez, 2005, p. 42) dice:

El reto del liderazgo es crear la arquitectura social de las ideas, las relaciones humanas, y la aventura florezca... Las ideas son la base para el cambio, para la reinvención, y para el capital intelectual... Las relaciones humanas tienen que ver con gente sobresaliente trabajando en armonía y apertura, donde todos se sienten empoderados, donde todos los colaboradores se sienten partícipes, competentes, significativos y en el centro de los acontecimientos. Y la aventura tiene que ver con el riesgo, con un prejuicio hacia la acción, con curiosidad y coraje.

Sobre el impacto del liderazgo para mejorar el rendimiento del estudiantado, Alemán (citado por Argos y Ezquerra, 2013, p.196) plantea que:

...para ejercer un buen liderazgo escolar se debe poseer una serie de valores y cualidades personales y humanas relacionadas con 
la flexibilidad, la institución, la facilidad para la negociación, la diplomacia, don de gentes, empatía y asertividad, inteligencia emocional, actitud positiva, disponibilidad, espíritu de sacrificio, vocación de servicio, capacidad de análisis y de síntesis, autodisciplina y domino del estrés.

Las citas anteriores se relacionan con el liderazgo y la motivación, porque si se quiere una educación de calidad para la niñez y juventud, se debe trabajar con humanismo, solidaridad, ética, innovación, creatividad y criticidad. Cada uno de los aspectos citados se logra cuando se trabaja con motivación. Cuando este aspecto se presenta, se tendrá una visión de un centro educativo de excelencia, se tendrá como meta principal el aprendizaje del estudiantado y el del mismo personal docente porque el aprendizaje es mutuo. El segundo siempre aprende algo nuevo. La motivación despierta los valores éticos que son importantísimos para construir el bienestar de la sociedad. Se crea la reflexión y criticidad para afrontar los diferentes problemas sociales. Se produce la realimentación entre el dirección, docente y estudiantes.

\section{Consideraciones finales}

Para finalizar, se hará una recapitulación con las preguntas que se plantearon al inicio. El liderazgo se debe ejercer en el siglo XXI con comunicación asertiva, escucha y motivación. Las tres son condiciones indispensables para llevar a cabo un buen trabajo y van concatenadas con una infinidad de aspectos como habilidades, destrezas, conocimientos, valores y actitudes. Todo en forma positiva para el beneficio de la educación, del desarrollo humano y de la sociedad.

La segunda pregunta se refiere a los desafíos que hay que vencer. En realidad, son los mismos: la asertividad, la escucha y la motivación. $\mathrm{Si}$ en un centro educativo entre estudiantes, directores o directoras, docentes, padres y madres de familia y la comunidad en general se presentan esas características, la calidad educativa sería excelente. Claro, esas condiciones de comunicación, escucha y motivación no siempre se cumplen, pero si se llegaran a cumplir sería un gran reto para no solo a nivel educativo sino a nivel de empresa u organización.

¿Por qué el liderazgo académico es importante en el siglo XXI? Ese el tercer cuestionamiento que se planteó al inicio de este ensayo. 
A lo largo del desarrollo de estas páginas, se recalca que el liderazgo académico es importante porque mejora el rendimiento académico, despierta el entusiasmo, la motivación, la comunicación asertiva, hay un mejor trabajo en equipo, el cual lo forman docentes, estudiantes y dirección. Si esta estrategia se logra, se creará un ambiente agradable no solo para el centro educativo, sino para el país porque se están formando las futuras generaciones constructoras de la nación.

Cuando se habla de liderazgo académico se refiere al sistema educativo que se inicia desde materno, pasa por el kínder, continúa con la escuela, la secundaria y finaliza con la universidad.

Después, la relación del liderazgo con el contexto educativo tiene mucha semejanza con los planteamientos citados. Si hay liderazgo, la educación será de mejor calidad, habrá mayor motivación, comunicación asertiva, reflexión y entusiasmo; en otras palabras, habrá un mejor proceso de enseñanza aprendizaje, lo cual implica un mejor desarrollo humano, social y educativo.

Por último, entre desafíos del siglo XXI está poder hacerle frente a los problemas de comunicación asertiva y a la motivación no solo en el centro educativo sino a nivel de familia, organizaciones, Gobierno y la sociedad en general. Si se lograra trabajar de mejor manera, esos temas, los problemas de violencia, corrupción, drogas, rendimiento académico, relaciones interpersonales en el aula, mejorarían en gran medida en todos los ámbitos, ya que las situaciones que nacen en el seno de la familia, a veces, continúan en los centros educativos.

\section{Referencias}

Calderón, M. (2010). Estudiante le dispara a directora en la cabeza. Periódico al Día. Recuperado de http://wvw.aldia.cr/ad_ee/2010/ julio/02/nacionales $2432032 . \mathrm{html}$

Civila, A. (2013). El ejercicio de liderazgo de los delegados/as de clase. En J.Argos y P. Ezquerra (Coords.), Liderazgo y educación (p.129-131). España: Editorial Universidad de Cantabria.

Cortés, A. (2004). Estilos de liderazgo y motivación laboral en el ambiente educativo. Revista Ciencias Sociales Universidad de Costa Rica, 106, 213. 
Elizondo, M. (1999). Asertividad y escucha activa en el ámbito académico. México: Editorial Trillas.

García, J. (2005). Liderazgo y educación. Revista del Humanismo Euroamericano, $18,35-69$.

Gutiérrez, M. (2001). El administrador educativo como líder académico. Revista Educare, 1, 37-49.

Gutiérrez, M. (2005). Liderazgo visionario y calidad de la educación en una época de retos y oportunidades. Revista Aportes, 1, 16-20.

Luna, M. (s. f.). El papel de líder en los procesos de cambio (Monografía). Recuperado de www.monografías.com/trabajos83/ papel-lider-procesos-cambio/papel-lider-procesos-cambio. shtml\#liderazgoa

Llorente, E. (2016). 6 formas de ser más efectivo. Emociones básicas. Recuperado de http://emocionesbasicas. com/2016/03/14/6-formas-de-ser-mas-asertivo/

Marchesi, A. y Martín, E. (2014).Calidad de la enseñanza en tiempos de crisis. Madrid: Editorial Alianza.

Programa de las Naciones Unidas para el Desarrollo. (2015). Informe sobre desarrollo humano. Recuperado de http://www.undp.org/ content/undp/es/home/librarypage/hdr/2015-human-development-report.html 